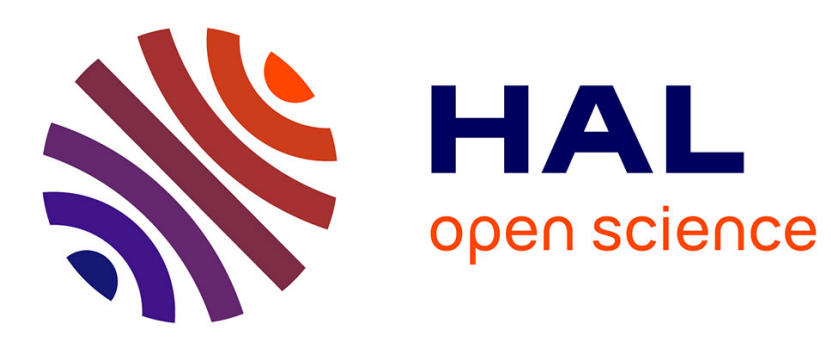

\title{
AUV (REDERMOR) obstacle detection and avoidance experimental evaluation
}

Isabelle Quidu, Alain Hétet, Yann Dupas, Stéphanie Lefèvre

\section{To cite this version:}

Isabelle Quidu, Alain Hétet, Yann Dupas, Stéphanie Lefèvre. AUV (REDERMOR) obstacle detection and avoidance experimental evaluation. IEEE OCEANS'07 conference, Jun 2007, Aberdeen (Scotland), United Kingdom. hal-00504875

\section{HAL Id: hal-00504875 \\ https://hal.science/hal-00504875}

Submitted on 22 Jul 2010

HAL is a multi-disciplinary open access archive for the deposit and dissemination of scientific research documents, whether they are published or not. The documents may come from teaching and research institutions in France or abroad, or from public or private research centers.
L'archive ouverte pluridisciplinaire $\mathbf{H A L}$, est destinée au dépôt et à la diffusion de documents scientifiques de niveau recherche, publiés ou non, émanant des établissements d'enseignement et de recherche français ou étrangers, des laboratoires publics ou privés. 


\title{
AUV (REDERMOR) OBSTACLE DETECTION AND AVOIDANCE EXPERIMENTAL EVALUATION
}

\author{
I. Quidu ${ }^{(1)}$, A. Hétet ${ }^{(2)}$, Y. Dupas ${ }^{(2)}$ and S. Lefèvre ${ }^{(3)}$
}

\begin{abstract}
Autonomous Underwater Vehicles (AUV) are expected to perform survey missions in both known and unknown environments. While the primary mission of an AUV is data collection, generally achieved with a sidescan sonar or a multibeam echosounder, another key task is to guaranty its own security. This paper deals with the problem of obstacle detection and avoidance by means of a forward looking sonar (FLS) mounted on the GESMA Redermor experimental AUV.
\end{abstract}

Index Terms - AUV, forward looking sonar, underwater object detection and avoidance, sonar target recognition.

\section{INTRODUCTION}

$\mathrm{W}$ hile the primary mission of an AUV is data collection, generally achieved with a sidescan sonar or a multibeam echosounder, another key task is to guaranty its own security. For that, the AUV needs to know in advance its environment and to find potential obstacles.

An "obstacle detection and avoidance" system is divided in a number of modules, each with its associated complexity (sensor, signal and information processing, vehicle behaviour). Each module can vary from one AUV to another, according to its size, its value and the use that one wants to make. Decisional autonomy is of primary importance for the definition of such a system. The AUV needs to process automatically information extracted from sonar images in order to choose own action strategies. Processing steps performed on FLS images can be as follows: 1) automatic detection of echoes and/or acoustic shadows; 2) object tracking and false alarm reduction; 3 ) echoes association and acoustic shadow characterization in order to estimate the shape or, at least, the extend of the obstacle; 4) rough localisation of obstacles to inform the vehicle supervisor in real-time about the position of the hazard; 5) bounding box definition around the detected obstacle defining an area to avoid; 6) selection between hazardous obstacles (net,

\footnotetext{
${ }^{(1)}$ Laboratoire E3I2 EA3876, Ecole Nationale des Etudes et Techniques de l'Armement - 2, rue François Verny, 29806 Brest Cedex 9, France. Phone : 332983489 15. E-mail: \{isabelle.quidu@ensieta.fr\}

(2) Groupe d'Etudes Sous-Marines de l'Atlantique, DGA/DET/GESMA, BP 42 - 29240 Brest Armées, France. Phone : 332982286 00. E-mail: \{alain.hetet,yann.dupas\}@dga.defense.gouv.fr

(3) Institut National des Sciences Appliquées - 135, Av. de Rangueil, 31077 Toulouse, France. E-mail: \{stlefevre@gmail.com\}
}

obstruction in the water column such as a tethered obstacle, unexpected escarpment, wreck, or other underwater structure), or harmless obstacles (fish groups, seaweeds).

The paper has been divided into three main parts. Firstly, it presents in Chapter II "Obstacle Detection and Avoidance" experiments performed at sea with a Reson Seabat 8101 Forward Looking Sonar (FLS) mounted with a depression angle of $15^{\circ}$ from the horizontal plane. The aim of this campaign was to record sonar data in several modes with various obstacles. Sonar data processing is then performed on FLS images sequences and described in chapter III. The technique is based on echo and shadow areas extraction. Image processing consists in thresholding and pattern recognition processing. The method for obstacle characterization is described in Chapter IV. Based on the previous results, a hazardous area is defined. It is based on a rough classification, obstacle tracking and bounding volume definition around the obstacle. Finally, avoidance strategies are discussed.

\section{OBSTACLE AND AVOIDANCE EXPERIMENT}

\section{A. Avoidance detection}

A Forward Looking Sonar (FLS) can prevent the vehicle from obstacles or terrain that may endanger the underwater vehicle. The AUV can be met with moored platform in harbors, reefs, fishes... A number of technologies have been developed during the last decade to perform obstacle avoidance [1], starting from the basic echo-sounder and going until the 3D high resolution sonar using a 2D planar array. Emphasis on this problem has been pointed out during the European MAST ASIMOV project [2]. However, looking for a robust detection and avoidance capability for AUV is still a hot topic today.

\section{B. Redermor 2 Architecture}

GESMA [3] has equipped its Redermor AUV with a network of 10 Tritech echosounders and a Reson Seabat 8101 Forward Looking Sonar. 


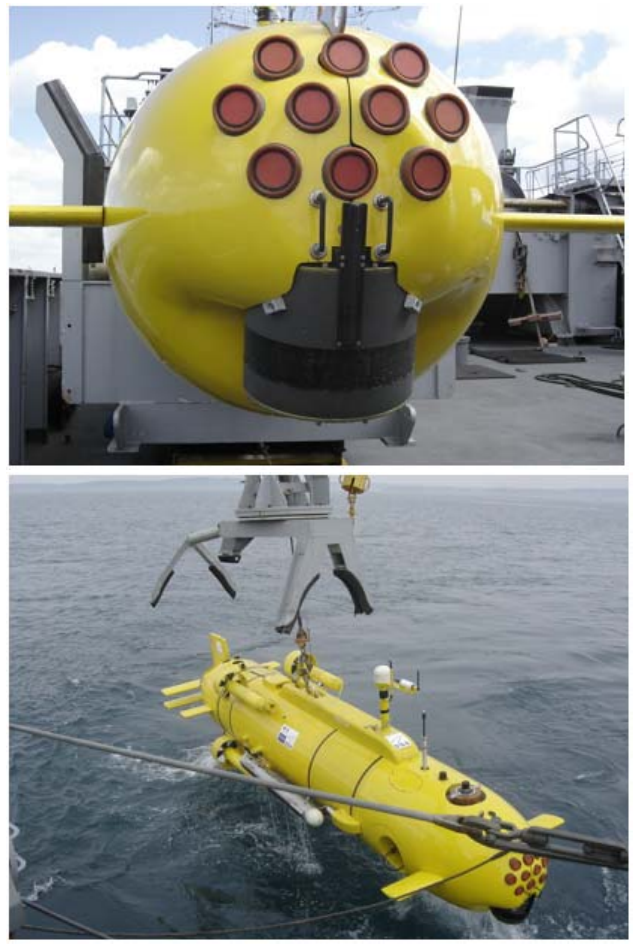

Fig. 1. Redermor vehicle: at the top, front view with the 10 echosounders network and the Reson 8101 FLS - at the bottom, one can see on the right of the vehicle the Klein 5400 side scan sonar on the Redermor size

The Redermor is the experimental platform deployed from the French Navy ship BEGM Thetis. It is a heavy and large AUV (3.8 tons x $6 \mathrm{~m}$ ). Navigation is performed knowing data from a Doppler Velocity Log (DVL) and a Motion Reference Unit (MRU). A Klein 5400 high resolution Side Scan Sonar (SSS) gives an acoustical imaging capability with a $20 \mathrm{~cm}$ azimuth resolution at $75 \mathrm{~m}$. Each echosounder of the network, working at $200 \mathrm{kHz}$ central frequency, operates over a $10^{\circ}$ horizon (at $-3 \mathrm{~dB}$ ). The echosounders are mounted in such a way that the main lobes are joined. They can be controlled individually, sequentially or by groups. The $240 \mathrm{kHz}$ Reson Seabat 8101 FLS is derived from a multibeam echosounder and can operate in bathymetric mode or a sector scan mode [4]. The system integrated in the Redermor can play a beamformed image over a $15^{\circ}$ (vertical) x $60^{\circ}$ (horizontal) sector with a $1.5^{\circ}$ azimuth resolution and a $5 \mathrm{~cm}$ range resolution. The sonar has been oriented $15^{\circ}$ from the horizontal plane.

\section{DEVITOBS'06 experiment}

In order to test the capability of the Redermor vehicle to react when obstacles are encountered on its way, GESMA organized an experimental trial in April 2006, named DEVITOBS'06 "DETection et EVITement d'OBStacles". The aim of this campaign was to record sonar data in several modes with various obstacles. In that way, it has been possible to test, qualify and upgrade the sensor suite, to initiate an obstacle database, to start algorithm development on those obstacles and to prepare avoidance tactics and strategies to be given to robot mission supervisor.

The experiment has been conducted in the Douarnenez Bay, near Brest. Several objects have been laid: a tethered mine like object, a net and plastic chains. Other objects have been investigated like the shipwreck "Meuse" in the same bay and schools of fish. Up to now analysis has been mainly focussed on the Reson 8101 data.

\section{SONAR DATA PROCESSING}

\section{A. Data characteristics}

The Reson Seabat 8101 FLS provides data that are processed and plotted on a sector Plan Position Indicator (PPI) display. This image is characterized by low contrast. The receive beam of $1.5^{\circ}(\mathrm{H})$ by $15^{\circ}(\mathrm{V})$ does not provide precise details and precise localization of the obstacle. For a range from 20 to $100 \mathrm{~m}$, the resolution cell increases from about $50 \mathrm{~cm}$ to $2.5 \mathrm{~m}$ in length according to the sector formed. The first step is to provide robust algorithms in order to avoid any obstacle. In addition to robustness, the implemented algorithms have to be low computable.

In the following, we make the assumption that only an obstacle exists ahead the vehicle.

\section{B. Image processing}

A segmented image of four classes can be derived from the raw sonar image. Each class is related to a specific area: strong echo area, medium echo area, background area and shadow area, from the stronger level to the lower level of pixels. In order to keep any potential alarm, strong echo detection simply consists in thresholding the sonar image. The threshold value is equal to a portion of the maximum level of the pixels, typically $75 \%$. This level is essential to keep valuable echoes.

The two following thresholdings are then applied to the low-pass filtered image. An average filtering is applied in order to smooth, hence to filter noise and to lower false alarm rate. The size of the filter mask takes into account sonar image resolution: finer in range than in azimuth. This is done to ensure an isotropic filtering within the meaning of ground scale. As a consequence, the mask size is minimal along the azimuth axis in order to preserve details and much larger along the range axis (according to the range to azimuth resolution sizes ratio). This filtered image is thresholded to extract shadow areas. This threshold is computed from the estimation of the reverberation mean [5]. For our bad contrasted images, threshold value equals to the estimated reverberation mean minus $3 \mathrm{~dB}$. At last, medium echoes detection is performed on the filtered image in the same way that previously for strong echoes.

\section{Human Machine Interface (HMI) development}

A HMI has been developed to simultaneously visualize both the raw and the segmented image. The interface also displays all figures and extracted obstacle features.

Two other images are designed to allow punctual echo tracking both in range and in azimuth. The first one is the classical echogram used by sonar operators in mine warfare: at 
each ping, we only keep the maximum pixel level along each line of the sonar image. We do the same along the other axis to form the second image: namely the original echogram. When a potential obstacle is detected, we extract locally the relative part of the image in the zoom image. By default or when no obstacle stands in sight, this last image is a zoom of the central part of the sonar image.

At last, some obstacle features can be computed from the segmented image and displayed: distance between the sonar and the obstacle, extent, height and also length of the obstacle, as well as A-scan plot of the echo if necessary.

\section{Results}

\section{1) Image segmentation}

We give here some results for two large obstacles: a wreck (or a seabed structure) and a school of fish.
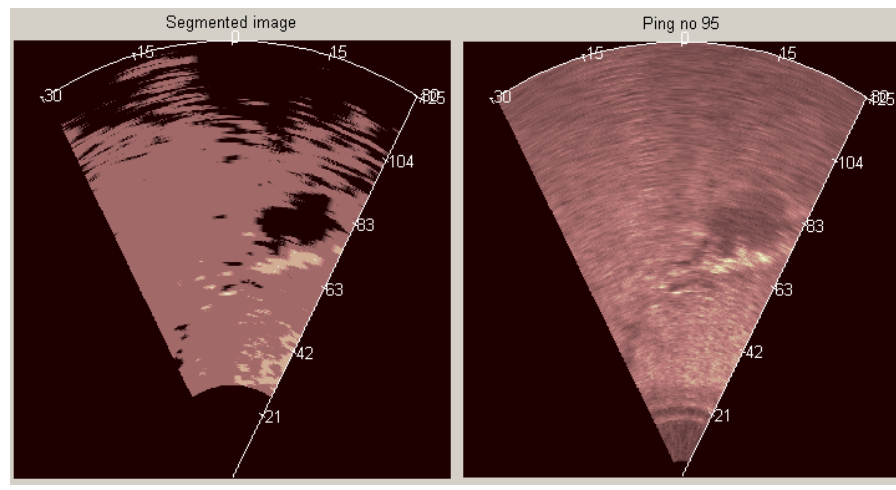

Fig. 2. sonar image of the wreck "Meuse" on the right and corresponding segmented image on the left
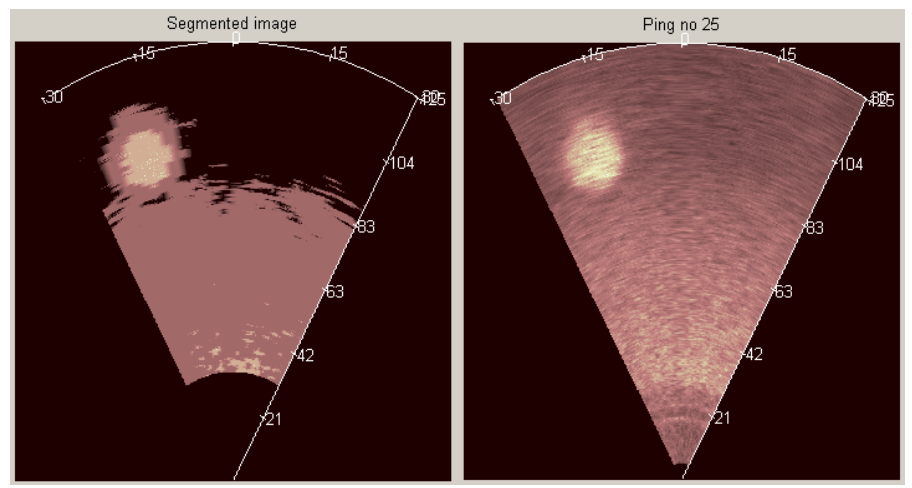

Fig. 3. sonar image of the school of fish on the right and corresponding segmented image on the left

2) HMI example

Fig. 4 shows a screenshot of the HMI in the case of the first class : tethered mine with a tether length of $5 \mathrm{~m}$.

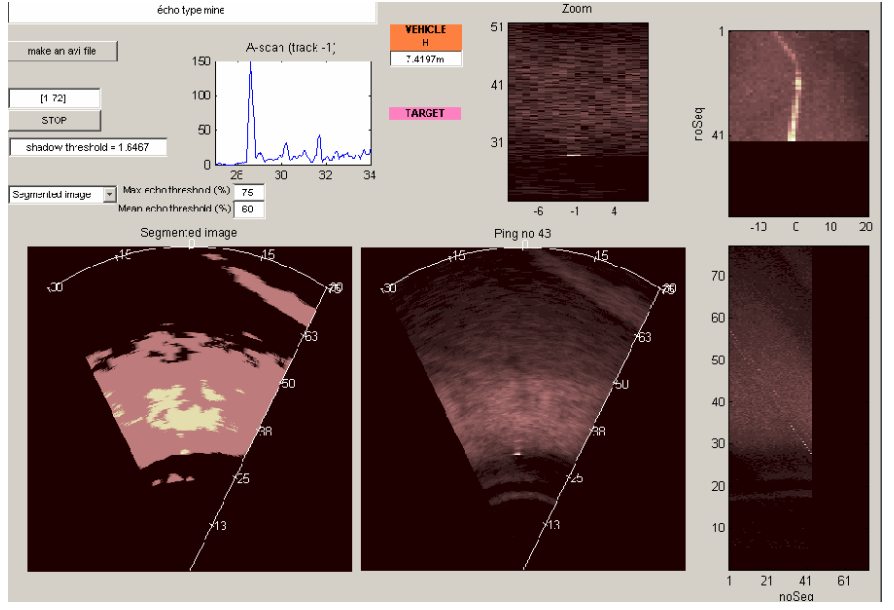

Fig. 4. HMI example

\section{OBSTACLE CHARACTERIZATION}

In this part we have made the hypothesis that the AUV is traveling above a flat seabed without any rough relief. We consider three obstacle classes: point target (labeled class 1), school of fish (labeled class 2) and shipwreck (labeled class $3)$.

\section{A. Single ping detection and rough classification of large obstacle}

We first perform a rough classification based on information available on images displayed on the HMI for a single ping.

The segmented image (excepted pixels corresponding to the water column and pixels located in the last noisy part of the image) presents several connected segmented areas whose pixels are labeled "echo" or "shadow". The largest area is then studied. If pixels which belong to this area are labeled "echo", we assume that the vehicle is ahead a potential school of fish (class 2). On the contrary, if a "shadow" structure is observed, and if we can find an "echo" area in front of the shadow, we assume that the vehicle is ahead a potential shipwreck or a seabed structure (class 3). Nevertheless, for these two cases, the contrast between this area and the background must be strong enough.

\section{B. Tracking of a still obstacle}

A still obstacle (of class 1 or class 3 ) can be tracked through the sequence. We have developed a tracking procedure performed on the sonar image by taking into account navigation information. An obstacle is visible on the sonar image thanks to the echo. Obstacles of the third class presents extended echo areas. On the contrary, for a point target of the first class, the tracking performs well by taking advantage of both the classical echogram and the original echogram where we can see tracks of the punctual echo. Detection of obstacles of the first class occurs only if no obstacle of the third class has been detected and roughly classified as such. We say that a point target is detected if it has been found and tracked on three consecutive pings.

As soon as a still obstacle is detected, the absolute position (latitude, longitude, depth) of the centroid of its echo is 
computed using both the information extracted from the image (relative position of the target) and the information about the current position of the AUV (longitude, latitude, depth, yaw, roll, pitch). At the next ping, we use the new position of the AUV and the absolute position of the obstacle detected on the previous image to compute the position that the obstacle should have on the new image if it has not moved. If we detect an obstacle in the vicinity of the new position and classify the same type of obstacle with sonar data gathered by the current ping, we consider that the same object has been detected in the two frames.

If an obstacle is tracked on at least three consecutive pings, we consider that it is not a false alarm. Another advantage of the tracking is to gather relevant features on this potential danger which will be useful to the avoidance (see next paragraph).

\section{Bounding volume around the obstacle}

Another useful information can be extracted from the sonar pictures.

The features extracted from a picture can be used to generate a bounding volume for the obstacle. This bounding volume sets a secure area around the obstacle, which is necessary for the obstacle avoidance.

The bounding volume is generated using the polygonal bounding area of the echo of the obstacle on the sonar image.

The absolute position (longitude, latitude, immersion) of each of the four points of the polygon is computed, based on an ellipse which surrounds the echo area. Each point has a longitude, latitude and depth error margin by tacking into account both the vertical aperture and the beam width (see figure 5).

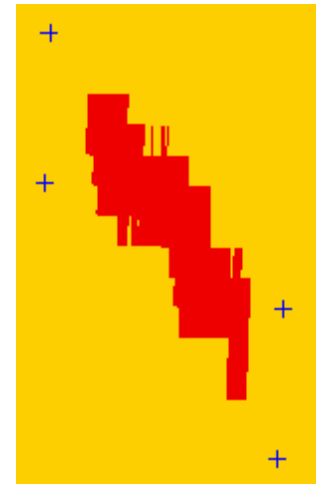

Fig. 5. Segmented echo area of a shipwreck and the 4 points delimiting a polygonal area around it

As a result we obtain thirty-two points (eight points for each of the four initial points as we can see on the figure 6). The eight points with the most extreme coordinates (longitude, latitude and depth), which demarcates the biggest volume, are consequently taken as corners of the final bounding volume.

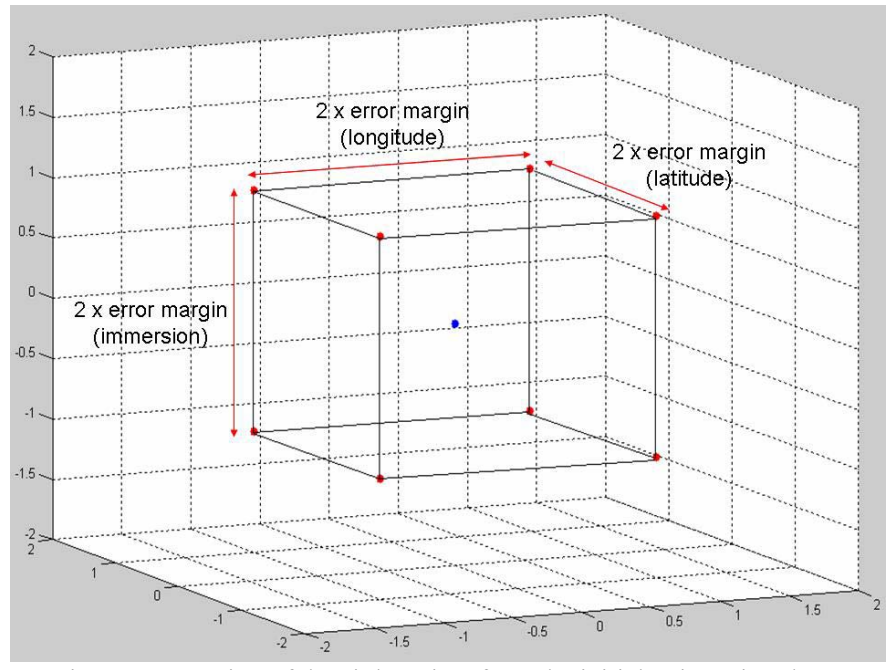

Fig. 6. Generation of the eight points from the initial point using the error margins

Obstacles of the third class (like shipwreck or seabed structure) have another feature that can be used to generate the bounding volume. The length of the shadow gives us an idea of height of the obstacle, thus we use this as the height of the bounding box, instead of the height of the volume previously processed. An example is given on the figure 7.

When an object is tracked through frames, a new bounding volume is generated each time. We chose to represent the bounding volume of an obstacle as the mean of all the bounding volumes generated since the first time it was detected.

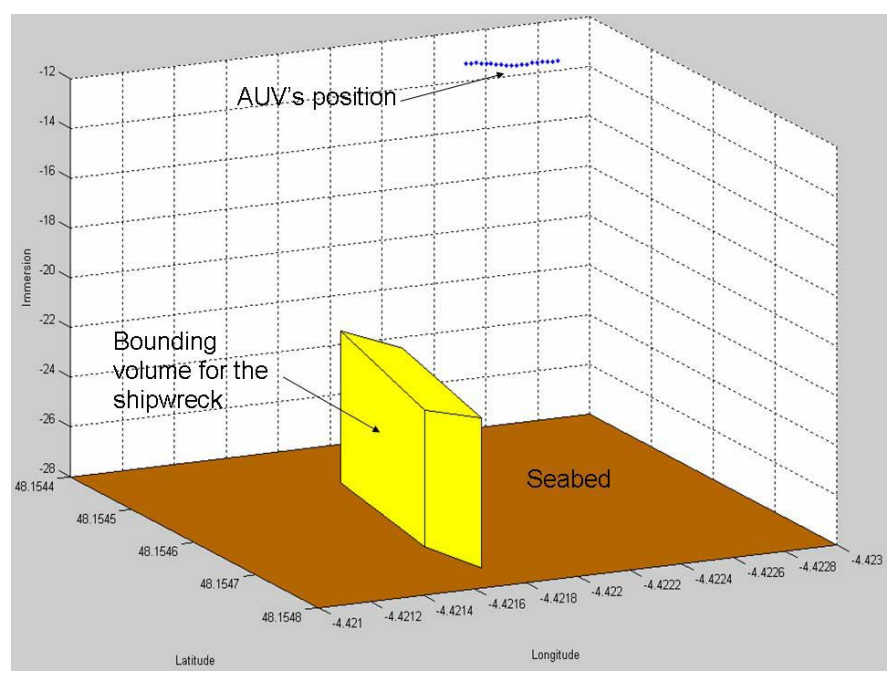

Fig. 7. Bounding volume of a shipwreck

We are now able to extract a simple geometric representation of an obstacle from successive sonar frames. This is a crucial information to enable the vehicle to decide what it will have to do next. 


\section{Avoidance Strategies}

\section{A. Context}

The information we obtained thanks to image processing together with navigation data gives to the AUV a partial knowledge of the encountered environment.

Depending on its mission and on the data collected on its neighbourhood, it must be able to react in order to keep its safety and to continue its operation.

As our approach is the use of AUVs in context of navy operations, we consider that the area where the AUV navigate is in essence dangerous, firstly because it can be in a badly known environment or because it can meet unexpected hazards.

Consequently, a great caution must be taken in the definition of the robot behaviour when it encounters an unpredicted echo. The use of a Forward Looking Sonar does not allow to conclude if the echo represents a real danger or not, but the way by which it can be analysed would depend on the AUV current mission.

\section{B. On transit mission}

Here, we consider that the AUV follows a sequence of waypoints, which have been determined manually (by a human operator), or automatically, using a global path planner algorithm.

The strategy commonly applied is to implement a reactive algorithm, like the potential field one [6] in order to directly avoid a potential collision with detected echoes.

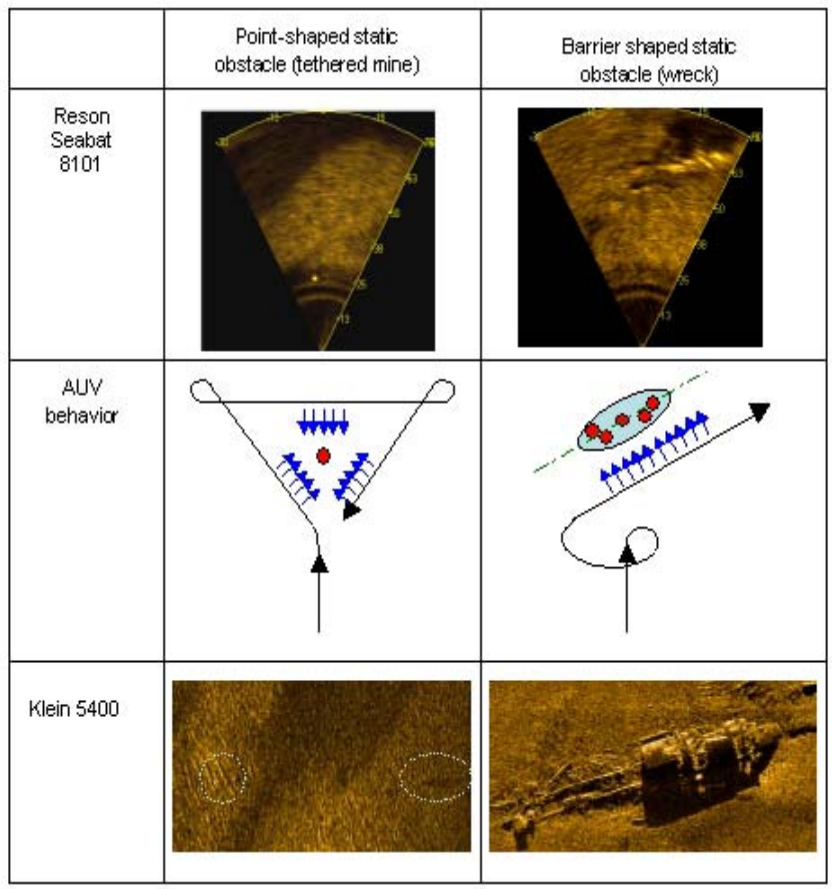

Fig 8 : Example of strategies for hazardous targets reacquisition with a high resolution SSS after FLS target detection. These examples are shown on static punctual obstacle and on a barrier shaped obstacle (shipwreck)

In our case, analysing these echoes could be a good way to improve our knowledge of the area we are working on. Moreover, if it is easy to avoid a punctual echo because of its reduced size, it is not the same problem with extended ones. Our example is on a shipwreck, but we can consider the fact that the obstacle is not completely seen by the sonar (object barrier).

Consequently, we think it would be interesting to get more information on the echo, for example with another sensor. FIG 8 shows some result obtained during the DEVITOBS'06 trials. It is obvious that data obtained with a high resolution side scan sonar allow a better classification of the echo. For object barrier, it will also allow to determine the shape and the limits of the echo, and a safe path to avoid it and go back on its first trajectory, using a local path planning algorithm.

If it appears that there is an important quantity of obstacles, it can be better to abort the mission, to consider the area as dangerous, and to reprogram the mission somewhere else.

\section{On exploration mission}

The problem is similar for exploration mission. For such operation, the AUV must cover different areas, generally with side scan sonar. This kind of operation is common to verify the clearness of other vehicles path.

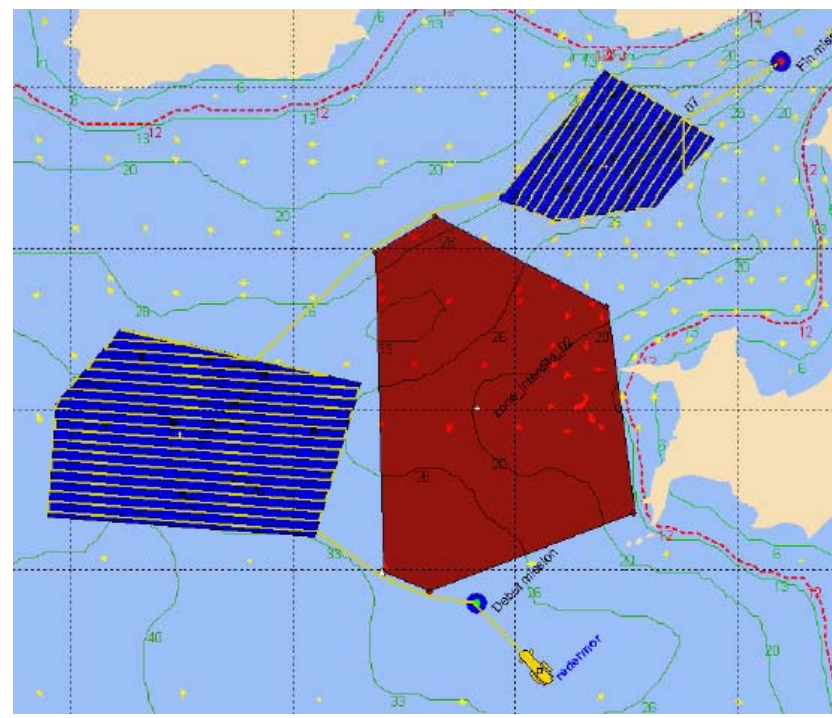

Fig 9 : Simulation of an automatic global path planning for exploration mission with two areas to explore (blue) and one to avoid (red) obtained with for Redermor AUV [7].

The first global trajectory can also be generated manually or by a global path planning algorithm (Fig 9). For such mission, it is important to follow precisely the programmed path, to be sure that the area has been correctly explored. Consequently, if the AUV detects an obstacle and tries to avoid it directly, it can create some holes in recorded data. Its avoidance behaviour must allow to:

- get more information on the obstacle, using other sensors (local planning) (Fig 10-b)

- define a security perimeter around the obstacle (Fig 10-b, c) - achieve the exploration of the zone, except the obstacle zone 
(global planning) (Fig 10-c)

As for transit mission, if the explored area appears to contain a high quantity of obstacles, it is better to stop the mission and to inform the operator of the situation.

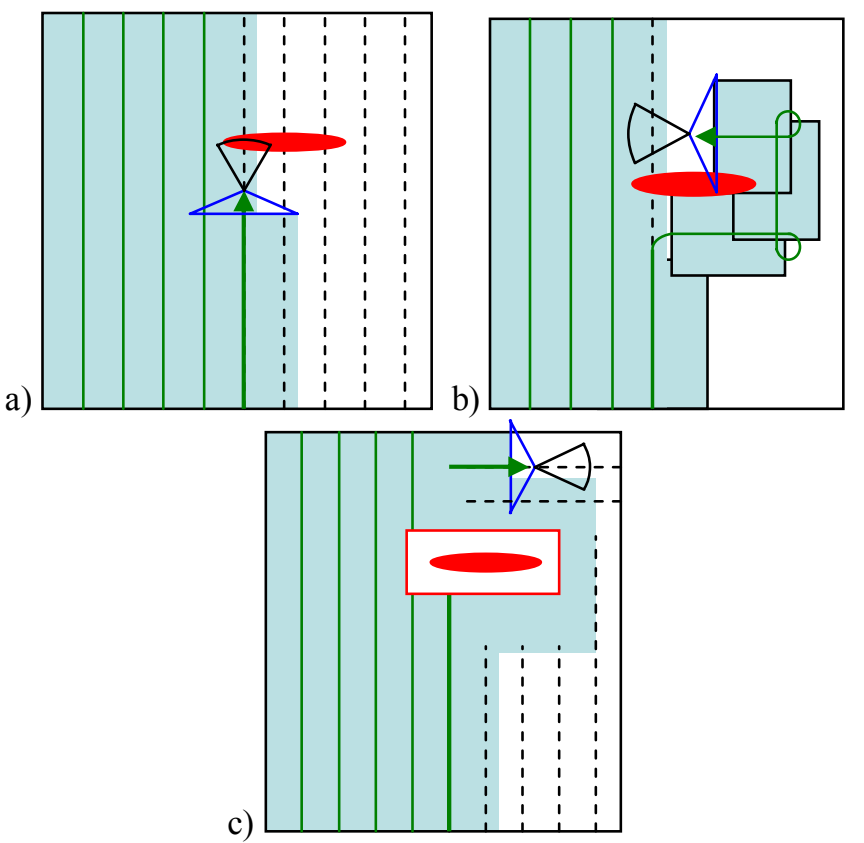

Fig $10(a, b, c)$ : Example of behavior to adopt when an obstacle is detected during an exploration mission. We represent the area covered by the SSS (blue) and the FLS (black): After the detection of an hazardous echo (red), the vehicle interrupt its mission (a), investigate the obstacle with a dedicated pattern (b), and continue the exploration of the area (c).

\section{CONCLUSION}

In this paper, algorithms have been developed to postprocess data from DEVITOBS'06 experiment using the GESMA testbed platform Redermor. More than an obstacle classification, we have shown that a bounding volume can be defined around the obstacle. This knowledge of the encountered environment of the AUV is of high interest to improve avoidance strategy capability.

\section{ACKNOWLEDGMENT}

The authors want to thank the GESMA Redermor team as the captain of the French Navy ship "Thétis" and its crew.

\section{REFERENCES}

[1] LOGGINS C.D., "A comparison of forward-looking sonar design alternatives", Proc. of MTS/IEEE Oceans'01, pp. 1536-1545, Honolulu, Hawaii, USA, 2001.
[2] PASCOAL et al, "Robotic Ocean vehicle for marine Science applications: the European ASIMOV Project", Proc. of MTS/IEEE Oceans'00, pp. 409-415, Providence, RI, USA, 2000.

[3] TOUMELIN N., LEMAIRE J., "New capabilities of the REDERMOR Unmanned Underwater Vehicle", Proc. of MTS/IEEE Oceans' 01, pp. 380-389, Honolulu, Hawaii, USA, 2001.

[4] CARPENTER R.N., MEDEIROS M.R., "Concurrent mapping and localisation and Map Matching on Autonomous underwater vehicles", Proc. of MTS/IEEE Oceans' 01, pp. 380-389, Honolulu, Hawaii, USA, 2001.

[5] JAN, D, «Traitement d'images numérique appliqué aux sonars à haute résolution », Proc. of GRETSI 87, Nice, France, 1987.

[6] KOREN Y., BORENSTEIN J., « Potential Field Methods and Their Inherent Limitations for Mobile Robot Navigation », Proc. of IEEE on Robotics and Automation, pp. 1398-1404, Sacramento, California, 1991.

[7] AYREAULT H. DABE F., BARBIER M., NICOLAS S., KERMET G., "Goal Driven Planning and Adaptivity for AUVs », Proc. of Control Architecture of Robots, Montpellier, FRANCE, April 2006. 